\title{
O uso de verbos em pré-escolares com desenvolvimento típico de linguagem
}

\author{
El uso de verbos en pre-escolares con \\ desarrollo típico del lenguaje
}

\section{The production of verbs in preschool children with typical language development}

\author{
Letícia Nóro* \\ Yasmin Alves* \\ Simone Nicolini de Simoni* \\ Paola Leonardi* \\ Fernanda Wietahn* \\ Helena Mota*
}

\section{Resumo}

Objetivo: analisar o uso dos verbos em crianças pré-escolares, com desenvolvimento linguístico típico, matriculadas na Educação Infantil da rede pública municipal de uma cidade do interior do RS. Métodos: a amostra foi composta por 48 crianças, 24 do sexo feminino e 24 do sexo masculino, com desenvolvimento linguístico típico e idades entre dois anos a cinco anos, divididas em oito grupos etários. Foram realizadas filmagens da fala espontânea de cada sujeito, e após, realizou-se a análise dos verbos, quanto ao tipo e ocorrência de todos os verbos produzidos por cada criança. Para análise estatística foram utilizados os testes de Kruskal-WalliseMann-Whitney, com nível de significância $p<0.05$. Resultados: não houve diferença no número de tipos verbais produzidos entre os sexos. Na comparação do número de tipos verbais produzidos entre as faixas etárias, houve diferença significativa em algumas faixas etárias, porém maiores valores na média da faixa de 4:7 a 4:11. Na totalidade da amostra foram produzidos 238 tipos verbais diferentes, sendo o verbo "ser" o de maior ocorrência. Conclusão: a quantidade de verbos

* Universidade Federal de Santa Maria, Santa Maria, Rio Grande do Sul, Brasil

Contribuição dos autores:

LN: concepção de desenho da pesquisa; YA: análise e interpretação dos dados, análise estatística e redação do manuscrito; SNS: análise e interpretação dos dados e redação do manuscrito; PL: análise e interpretação dos dados; FW: obtenção dos dados e redação do manuscrito; HM: revisão crítica do manuscrito quanto ao conteúdo intelectual importante.

E-mail para correspondência: Simone Nicolini de Simoni simonedesimoni@hotmail.com Recebido: $16 / 08 / 2017$

Aprovado: 02/04/2018 
produzidos não é influenciada pelo sexo das crianças. De modo geral, o uso de verbos aumenta conforme a idade, exceto nas faixas etárias de cinco anos, onde houve um pequeno decréscimo.

Palavras-chave: Fonoaudiologia; Desenvolvimento infantil; Pré-escolar; Linguagem; Vocabulário

\section{Abstract}

Objective: to analyze the use of verbs in pre-school children with typical linguistic development enrolled in the public Early Childhood Education in an inner city of RS. Methods: The sample consisted of 48 children, 24 females and 24 males, with typical linguistic development and ages between two and five years old; they were divided into eight age groups. Recordings were realized of the spontaneous speech of each subject. Afterwards, the verbs were analyzed by the type and occurrence for each child. For statistical analysis, the Kruskal-WalliseMann-Whitney tests were used, with significance level $\mathrm{p}$ $<0.05$. Results: There was no difference in the number of verbal types produced between the genders. Comparing the number of verbal types produced between the groups, there was a significant difference in some age groups, but higher values were found in the range of $4: 7$ to $4: 11$. A total of 238 different verbal types were produced in the sample, with the verb "to be" having the highest occurrence. Conclusion: the number of verbs produced is not influenced by the sex of the children. In general, the use of verbs increases with age, except in the five-year age group, where there was a small decrease.

Keywords: Speech, Language and Hearing Sciences; Child Development; Preschool; Language; Vocabulary.

\section{Resumen}

Objetivo: analizar el uso de los verbos en niños preescolares, con desarrollo lingüístico típico, matriculados en la Educación Infantil de la red pública municipal de una ciudad del interior del RS. Métodos: la muestra fue de 48 niños, 24 del sexo femenino y 24 del sexo masculino, con desarrollo lingüístico típico y edades entre dos y cinco años, divididas en ocho grupos erarios. Se realizaron filmaciones del habla espontánea de cada sujeto, y después, se realizó un análisis de los verbos, en cuanto al tipo y ocurrencia de todos los verbos producidos por cada niño. Para el análisis estadístico fueron utilizados las pruebas de Kruskal-WalliseMann-Whitney, con nivel de significación $p<0,05$. Resultados: no hubo diferencia en el número de tipos verbales producidos entre los sexos. En la comparación del número de tipos verbales producidos entre los grupos de edad, hubo diferencia significativa en algunas franjas etarias, pero mayores valores en la media de la banda de 4:7 a 4:11. En la totalidad de la muestra fueron producidos 238 tipos verbales diferentes, siendo el verbo "ser" el de mayor ocurrencia. Conclusión: la cantidad de verbos producidos no es influenciada por el sexo de los niños. En general, el uso de verbos aumenta según la edad, excepto en las franjas de edad de cinco años, donde hubo un pequeño descenso.

Palabras clave: Fonoaudiología; Desarrollo Infantil; Preescolar; Lenguaje; Vocabulario

\section{Introdução}

O presente artigo trata da aquisição dos verbos por pré-escolares com desenvolvimento linguístico típico, utilizando-se prioritária, mas não exclusivamente, a concepção inatista, segundo a qual a linguagem é o resultado da inter-relação do estado inicial (dispositivo de aquisição da linguagem) aliado ao curso da experiência. ${ }^{1}$

A aquisição dos verbos, segundo estudos, proporciona o desenvolvimento gramatical, pois sua função principal é atuar como predicado, expressando ações; seu emprego ocorre precocemente, por volta dos dois anos de idade, sendo aprimorado entre o terceiro e quarto ano de vida. . $3,3,4^{2}$

Sabe-se, de acordo com a literatura, que há diferenças entre a aquisição de verbos e substantivos. Os substantivos geralmente são adquiridos primeiro devido à sua forma mais concreta, o que não quer dizer que as crianças imediatamente compreendam o significado pleno da palavra, mas, a representação inicial é suficientemente concreta para incluir 
outros membros da mesma classe gramatical; além disso, os verbos são termos relacionais, referem-se a conceitos mais abstratos e menos coesos. Consequentemente, os limites que diferenciam um verbo de outro são menos claros e de mais difícil aprendizado. ${ }^{5,6}$

A aquisição de verbos pode apresentar dificuldades por haver diversidade semântica, ou seja, a atribuição do significado à palavra. Também, a questão gramatical, por se direcionar às regras sintáticas. Sendo assim, a aquisição de verbos pode sofrer comprometimento para a generalização, fazendo com que a criança necessite ser apresentada ao verbo mais vezes para internalizar suas prioridades. ${ }^{7,8}$

A aquisição das palavras é fator primordial para a comunicação, há a expressão denominada de "explosão do vocabulário" que denota o período do aumento de vocábulos, entre eles, os verbos. De acordo com a literatura compulsada, há verbos que são referentes às ações físicas, como por exemplo, puxar, andar, e há verbos classificados como estados mentais, por exemplo, querer, pensar, ser, estar, entre outros. Os verbos relacionados às ações físicas são reconhecidos facilmente durante o período de aquisição, quando comparado aos verbos mentais. $^{8}$

Essa particularidade de aquisição dos verbos pode ser observada no momento da apresentação, ou seja, antes ou após o resultado da ação; os verbos de resultado são favorecidos pela condição completa (verbo após a ação), e os verbos de movimento favorecidos pela condição futura (verbo antes da ação). Assim, verbos com referência a ação física geram combinações de memória mais fortes, sendo acessados mais facilmente que verbos simples. Além disso, quanto mais um verbo for estimulado, mais frequentemente, e com maior flexibilidade, ele aparecerá na fala da criança, o que indica que o refinamento de seu conhecimento ocorre por meio do uso. ${ }^{9,10}$

Enfim, com os conceitos de tempo e aspecto verbal a criança poderá construir representações acerca do predicado, pensar sobre os eventos e assim ter elementos para a construção de um sistema linguístico, sendo esses conceitos fundamentais para a aquisição da linguagem. ${ }^{8}$

A partir do exposto e considerando a importância da aquisição dessa classe gramatical para o desenvolvimento da linguagem, o objetivo deste estudo é analisar o uso dos verbos em amostras de fala espontânea de crianças pré-escolares comparando à variável gênero feminino e masculino, com desenvolvimento típico de linguagem.

\section{Métodos}

Esta pesquisa é de caráter quantitativo e descritivo, a coleta de dados é considerada de caráter transversal, fazendo parte de um projeto aprovado no Comitê de Ética da instituição de origem sob o número 0219.0.243.000-11. Para participação na pesquisa, os responsáveis pelos sujeitos assinaram o Termo de Consentimento Livre e Esclarecido - TCLE. Conforme consta no TCLE, uma cópia permanece com o sujeito e destina-se ao arquivo acadêmico do pesquisador, de acordo com a resolução 466/12 da Comissão Nacional de Ética em Pesquisa-CONEP/2012.

A amostra deste artigo foi selecionada por conveniência e é composta por 48 sujeitos pareados por sexo e idade com idades entre dois anos e cinco anos, 11 meses e 29 dias, separando-os entre 24 sujeitos do sexo masculino e 24 sujeitos do sexo feminino, distribuídos uniformemente entre faixas etárias de seis meses cada, conforme exposto no quadro a seguir. 
Quadro 1. Distribuição dos sujeitos pareados por sexo e idade.

\begin{tabular}{|c|c|c|c|}
\hline Faixa etária & Número de sujeitos & Sexo Masculino & Sexo Feminino \\
\hline $2: 00-2: 6$ & 6 & 3 & 3 \\
\hline $2: 6-2: 12$ & 6 & 3 & 3 \\
\hline $2: 12-3: 6$ & 6 & 3 & 3 \\
\hline $3: 6-3: 12$ & 6 & 3 & 3 \\
\hline $3: 12-4: 6$ & 6 & 3 & 3 \\
\hline $4: 6-4: 12$ & 6 & 3 & 3 \\
\hline $4: 12-5: 6$ & 6 & 3 & 3 \\
\hline $5: 6-5: 11: 29$ & 6 & 3 & 24 \\
\hline Total de sujeitos & 48 & 24 & 3 \\
\hline
\end{tabular}

Nos critérios de inclusão todas as crianças eram membros de famílias monolíngues falantes do Português Brasileiro (PB), com desenvolvimento típico de linguagem, considerando a aquisição fonológica típica e estratégias de reparo condizentes com a idade. Já como critérios de exclusão, considerou-se presença de perda auditiva, comprometimento neurológico, emocional e/ou cognitivo, detectável por meio de observação; presença de alterações motoras ou orgânicas orais, ou crianças que tivessem realizado/estivessem realizando fonoterapia.

A coleta dos dados foi realizada em escolas municipais de Educação Infantil de uma cidade do interior do estado do Rio Grande do Sul. Para realizar a avaliação fonoaudiológica incluiu-se o questionário destinado aos responsáveis (anamnese), avaliação orofacial (AMIOFE versão reduzida), avaliação das praxias orais, avaliação da linguagem oral por meio do Protocolo Observacional Comportamental (PROC) e triagem auditiva realizando a pesquisa dos limiares auditivos por via aérea nas frequências de $500 \mathrm{~Hz}, 1000 \mathrm{~Hz}, 200$ $\mathrm{Hz}$ e $4000 \mathrm{~Hz}$ testados a $20 \mathrm{~dB}$ NA, para verificar se há presença de alterações auditivas periféricas.

O questionário, respondido pelos responsáveis individualmente, foi adequado com aspectos sobre o desenvolvimento global da criança, além de aspectos relacionados à dinâmica familiar.

Para avaliação dos aspectos miofuncionais orofaciais empregou-se o "Protocolo de avaliação miofuncional orofacial com escores (AMIOFE)" "11 adaptado, foram analisadas as estruturas e funções do Sistema Estomatognático no que se refere ao aspecto, posição habitual, tensão muscular, mobilidade e respiração. Para as crianças com idade superior a três anos e seis meses, utilizou-se adicionalmente o Protocolo de avaliação da dispraxia ${ }^{12}$ para verificação das praxias orais. Utilizaram-se essas avaliações a fim de descartar comprometimento nesses níveis.

A avaliação da linguagem oral foi realizada por meio do "Protocolo de Observação Comportamental" " ${ }^{13}$ para as crianças até quatro anos. Com as crianças maiores, atentou-se para narrativas orais espontâneas, resposta a perguntas e observação do brincar. A coleta da fala espontânea para crianças acima de 4 anos foi feita sem o uso do protocolo, visto que este é para crianças de até quatro anos, visto que a sequência dos procedimentos foram os mesmos para todas as crianças, com o uso dos mesmos brinquedos para crianças maiores de quatro anos, havendo interação entre pesquisador e criança para o direcionamento da atividade de diálogo, onde todos os trechos foram analisados.

Além disso, os aspectos fonéticos e fonológicos da fala foram observados em conversação espontânea e repetição de vocábulos foneticamente balanceados. Ressalta-se que essa avaliação foi prévia às filmagens de fala espontânea e foi utilizada apenas como um balizador do desenvolvimento de linguagem para incluir ou excluir as crianças do estudo.

A triagem auditiva para as crianças até dois anos, seis meses e 29 dias de idade foi realizada por meio da Audiometria de Reforço Visual ${ }^{14}$,e para as crianças na faixa etária de dois anos e sete meses a cinco anos, 11 meses e 29 dias foi realizada a avaliação audiológica, com audiometria lúdica condicionada ou audiometria tonal liminar. ${ }^{15}$ Caso houvesse falha nas respostas, em uma ou mais frequências, e em duas triagens consecutivas, a criança era encaminhada para avaliação otorrinolaringológica e audiológica completa. 
Assim, com todas as crianças que passaram pelos critérios de inclusão (incluídas nos dados deste artigo), realizou-se a avaliação do vocabulário expressivo por meio de fala espontânea $\mathrm{e}$ nomeação de objetos e brinquedos em miniatura em situação de brinquedo, sem a utilização de perguntas direcionadas.

Não foi realizada avaliação do vocabulário receptivo porque não era objetivo do estudo. Optou-se pela fala espontânea já que o objetivo do projeto de pesquisa ao qual este artigo pertence era analisar todas as classes gramaticais e os protocolos não contemplam esse critério, enfatizando a produção e compreensão de substantivos.

Foram realizadas filmagens das avaliações mencionadas acima durante 20 minutos com uma filmadora da marca Samsung, modelo SMX-C200, que posteriormente foram armazenadas em HD externo para transcrição fonética ampla da fala de cada criança e transcrição alfabética da fala da examinadora. Para a transcrição da fala das crianças até três anos e três meses, utilizou-se o método do consenso $^{16,17}$, ou seja, duas julgadoras trabalharam independentemente na transcrição; após, as transcrições foram comparadas e as discrepâncias ouvidas, novamente, por uma terceira julgadora até chegarem à concordância em todos os enunciados/ palavras/sons produzidos pela criança. Caso não houvesse a concordância entre pelo menos duas julgadoras, o trecho era excluído. Assim, garantiu-se a confiabilidade das transcrições, evitando que um grande número de palavras fosse excluído, já que crianças pequenas, mesmo com desenvolvimento típico apresentam maior variabilidade e ininteligibilidade nas produções.

Já para as crianças das demais faixas etárias, que apresentam as produções mais estáveis, utilizou-se o seguinte método de confiabilidade entre as transcrições: todas as amostras foram transcritas por uma julgadora experiente em linguagem infantil. Uma segunda avaliadora com a mesma experiência transcreveu, independentemente, $20 \%$ da mesma amostra para atestar a confiabilidade ${ }^{18,19}$. Assim, a média de concordância foi de 79,6\% para as faixas de 3 anos; $81,9 \%$ para as faixas de 4 anos e $80,1 \%$ para as faixas de 5 anos.

Para análise dos verbos, inicialmente, todas as palavras produzidas por cada criança foram listadas em planilha do Microsoft Excel; após, os verbos foram identificados e listados em nova planilha, obtendo-se todos os verbos produzidos (ocorrências) por cada criança. Então foram excluídos os verbos repetidos para contabilizar o número de verbos diferentes produzidos (tipos).

Ressalta-se que a nomenclatura "ocorrências e tipos" refere-se ao número total de palavras produzidas (neste caso, verbos) em oposição ao número de palavras diferentes produzidas (neste caso, contabilizou-se cada verbo diferente dito apenas uma vez).

Por fim, para a análise estatística, utilizou-se o Programa Statistical Analysis System, versão 9.2 para realização de tabelas de frequência quanto ao número de tipos e ocorrências dos verbos por faixa etária e dos verbos que foram mais produzidos no total da amostra (ocorrência superior a 50). Após, com o mesmo programa, utilizou-se o teste de Mann-Whitney para comparação dos tipos verbais produzidos entre os sexos e, o teste de Kruskal-Wallis para comparação dos tipos verbais entre as faixas etárias. Em ambos os testes, o nível de significância adotado foi de $5 \%(\mathrm{p}<0,05)$.

\section{Resultados}

Na Tabela 1 é apresentada a comparação do número de tipos de verbos produzidos entre os sexos. Observa-se que as meninas obtiveram maior média percentual, porém não houve diferença estatisticamente significante entre os sexos para os tipos verbais produzidos.

Tabela 1. Análise comparativa dos tipos verbais produzidos entre os sexos.

\begin{tabular}{cccc}
\hline Sexo & Número de sujeitos & $\begin{array}{c}\text { Média do número de } \\
\text { tipos verbais }\end{array}$ & Valor de $\boldsymbol{p}$ \\
\hline Feminino & 24 & 45,04 & $\mathrm{P}=0,239$ \\
Masculino & 24 & 40,79 & \\
\hline
\end{tabular}

Teste estatístico utilizado:Mann-Whitney; valor de significância: $\mathrm{p}<0,05$. 
A Tabela 2 mostra a comparação do número de tipos verbais produzidos entre as faixas etárias. O teste de Kruskal-Wallis evidenciou diferença significativa entre as faixas etárias para o número de tipos verbais produzidos.
A totalidade da amostra produziu 238 tipos verbais diferentes. Quanto às ocorrências verbais por sujeito houve variação de 43 (faixa etária de $3: 0-3: 6 ; 29)$ a 226 (faixa etária de 4:0 - 4:6;29), sendo a média geral de 124,13 ocorrências.

Tabela 2. Análise comparativa do número de tipos verbais produzidos entre faixas etárias

\begin{tabular}{ccc}
\hline Faixa etária & Média de tipos verbais produzidos & Valor de $p$ \\
\hline (A) $2: 0-2: 6 ; 29$ & 28,17 & \\
(B) $2: 7-2: 11 ; 29$ & 35,50 & \\
(C) $3: 0-3: 6 ; 29$ & 36,17 & \\
(D) $3: 7-3: 11 ; 29$ & 46,67 & \\
(E) $4: 0-4: 6 ; 29$ & 48,33 & \\
(F) $4: 7-4: 11 ; 29$ & 53,83 & \\
(G) $5: 0-5: 6 ; 29$ & 45,00 & \\
(H) $5: 7-5: 11 ; 29$ & 49,67 & \\
\hline
\end{tabular}

Teste estatístico utilizado: Kruskal-Wallis. Valor de significância: $p<0$,05. Quanto à significância, $A \neq D, E, F, G, H ; C \neq F$ e $B \neq F$.

Quanto à tipologia, o verbo mais produzido pelas crianças foi o "ser", com um total de 1240 ocorrências. Na Tabela 3 constam os verbos mais produzidos pelos sujeitos (ocorrência igual ou superior a 50 na totalidade da amostra).

Tabela 3. Verbos de maior ocorrência na amostra geral

\begin{tabular}{cc}
\hline Verbo & $\begin{array}{c}\text { Número de ocorrências } \\
\text { na totalidade da amostra } \\
(\mathbf{N}=\mathbf{4 8})\end{array}$ \\
\hline Ser & 1240 \\
Ter & 652 \\
Ir & 635 \\
Olhar & 419 \\
Estar & 318 \\
Saber & 269 \\
Achar & 120 \\
Ver & 115 \\
Dar & 108 \\
Fazer & 104 \\
Querer & 91 \\
Deixar & 81 \\
Botar & 77 \\
Pegar & 75 \\
Ficar & 73 \\
Cair & 73 \\
Comer & 62 \\
Poder & 59 \\
Andar & 50 \\
\hline
\end{tabular}

Nas duas faixas etárias de dois anos, foram produzidos 67 e 78 verbos diferentes; nas faixas de três anos, 85 e 102 verbos diferentes; nas faixas de quatro anos, 94 e 111 verbos diferentes; finalmente, nas faixas de cinco anos, 101 e 112 verbos diferentes, respectivamente. A Tabela 4 apresenta os cinco verbos de maior ocorrência por faixa etária.

Ressalta-se que se utiliza a nomenclatura " $\mathrm{ti}$ pos verbais" quando a referência é ao número de verbos diferentes produzidos. Ou seja, cada verbo foi contabilizado apenas uma vez, não contando no cálculo suas repetições. 
Tabela 4. Verbos de maior ocorrência por faixa etária

\begin{tabular}{|c|c|c|}
\hline Faixa Etária & Verbo & Número de ocorrências \\
\hline \multirow{5}{*}{$2: 0-2: 6 ; 29$} & Ser & 119 \\
\hline & Ter & 52 \\
\hline & $\mathrm{Ir}$ & 45 \\
\hline & Estar & 28 \\
\hline & Cair & 2 \\
\hline \multirow{5}{*}{$2: 7-2: 11 ; 29$} & Ser & 101 \\
\hline & $\mathrm{Ir}$ & 86 \\
\hline & Ter & 77 \\
\hline & Estar & 43 \\
\hline & Olhar & 31 \\
\hline \multirow{5}{*}{$3: 0-3: 6 ; 29$} & Ser & 128 \\
\hline & Olhar & 76 \\
\hline & $\mathrm{Ir}$ & 68 \\
\hline & Ter & 61 \\
\hline & Estar & 39 \\
\hline \multirow{5}{*}{$3: 7-3: 11 ; 29$} & Ser & 136 \\
\hline & $\mathrm{Ir}$ & 97 \\
\hline & Ter & 72 \\
\hline & Olhar & 65 \\
\hline & Estar & 51 \\
\hline \multirow{5}{*}{$4: 0-4: 6 ; 29$} & Ser & 134 \\
\hline & Ir & 109 \\
\hline & Ter & 92 \\
\hline & Olhar & 80 \\
\hline & Estar & 50 \\
\hline \multirow{5}{*}{$4: 7-4: 11 ; 29$} & Ser & 256 \\
\hline & Ter & 111 \\
\hline & $\mathrm{Ir}$ & 95 \\
\hline & Saber & 83 \\
\hline & Olhar & 67 \\
\hline \multirow{5}{*}{$5: 0-5: 6 ; 29$} & Ser & 200 \\
\hline & Ter & 80 \\
\hline & Saber & 61 \\
\hline & $\mathrm{Ir}$ & 54 \\
\hline & Olhar & 50 \\
\hline \multirow{5}{*}{$5: 7-5: 11 ; 29$} & Ser & 166 \\
\hline & Ter & 107 \\
\hline & Ir & 81 \\
\hline & Saber & 52 \\
\hline & Olhar & 31 \\
\hline
\end{tabular}

\section{Discussão}

Inicialmente, verifica-se que a produção do número de tipos verbais não foi afetada pelo sexo, indicando assim, que as influências nesse processo mostram-se mais pela própria maturação linguística, típica do desenvolvimento e pela interação comunicativa em que a criança está inserida do que em relação ao sexo. ${ }^{20,21,22}$
Esses achados corroboram a um estudo que teve como objetivo verificar a diversidade quantitativa e qualitativa dos verbos enunciados por crianças com idades entre 2 e 4 anos falantes do Português Brasileiro, bem como sua evolução, em que também não houve diferença estatística entre os sexos. ${ }^{4}$

A comparação dos tipos verbais conforme a faixa etária, observada na Tabela 2 , evidencia a evolução da produção dos verbos conforme a 
idade. Isto pode ser explicado devido ao maior tempo de exposição que os últimos sujeitos tiveram à língua que estão adquirindo, apresentando maior estabilidade. $\mathrm{O}$ mesmo pode ser encontrado em uma pesquisa que realizou um experimento, investigando a influência sintática como recurso ativo durante o processo de aquisição que estreita as possibilidades de formulação de hipóteses das crianças sobre o significado dos novos verbos. ${ }^{22}$

Quanto à quantidade, pode-se afirmar que, de modo geral, um grande número de verbos diferentes foi produzido na amostra, tanto considerando a amostra total quanto por faixas etárias. Outros estudos sobre a aquisição de línguas germânicas também verificaram alta produtividade de verbos, com crescimento linear e maiores incrementos durante o terceiro e quarto ano de vida ${ }^{22,23}$, semelhante ao que ocorreu no presente estudo, exceto em relação à linearidade, já que aqui foram encontradas algumas regressões nas faixas etárias de cinco anos. Estudo brasileiro também encontrou linearidade na aquisição dos verbos em crianças de dois a quatro anos de idade. ${ }^{4}$ É possível que a regressão tenha ocorrido por dois motivos: um seria o maior desenvolvimento da sintaxe nessa idade e outro, a consciência das trocas fonológicas ainda presentes. Ambas as situações exigem mais do processamento linguístico, podendo levar a desequilíbrios em outras áreas, como o vocabulário, especificamente, no uso de verbos. ${ }^{24}$

Quanto aos verbos mais prevalentes no vocabulário das crianças, há semelhanças com um estudo longitudinal, já que a maioria dos verbos são os mesmos nos dois estudos. ${ }^{25}$ Além disso, a autora afirma que as formas verbais mais iniciais $\mathrm{e}$ recorrentes são o imperativo/ presente e o infinitivo, bastante similar aos achados desta pesquisa.

Outro artigo traz uma lista com os verbos mais produzidos por crianças entre dois e quatro anos de idade. ${ }^{4}$ Todos os verbos dessa lista estão dentre os que tiveram ocorrência superior a 50 na presente pesquisa, exceto o verbo "pôr", que foi aqui produzido como "botar", porém deve-se considerar que as diferenças socioculturais, podem vir influenciar na produção dos verbos "pôr" e "botar".

Tanto no presente estudo quanto em trabalhos semelhantes ${ }^{4,23}$, os verbos mais prevalentes foram praticamente os mesmos, o que se deve à frequência de estímulos tanto na língua quanto no ambiente em que a criança está inserida.
Dentre os verbos com maior ocorrência na amostra geral e por faixa etária, encontram-se tanto verbos que indicam ações físicas (ir, olhar, achar, etc.) quanto verbos que indicam estados mentais (ser, ter, estar, saber, etc.). Segundo um estudo ${ }^{7}$, os primeiros seriam de mais fácil aquisição por gerarem códigos de memória mais fortes, o que não foi confirmado pela presente pesquisa, dada a distribuição equilibrada de ambos na amostra. A maior ocorrência do verbo "ser" pode ser devido à função deste, que pode representar estados mentais, mas também é um verbo de ligação, sendo, portanto, muito frequente na maioria das sentenças.

\section{Conclusão}

É possível concluir que há uma prevalência do uso dos verbos nas faixas etárias maiores, demonstrando, de modo geral, evolução conforme a idade, exceto na faixa etária de cinco anos. Quanto aos verbos mais produzidos por sujeito encontrou-se produção de verbos diferentes, com predomínio do verbo "ser". Não houve diferença entre os sexos quanto à quantidade de verbos produzidos.

Esses resultados propiciam mais uma importante fonte de conhecimento sobre a aquisição da linguagem, especificamente em relação à produção verbal. Sugere-se, ainda, a realização de estudos com metodologias semelhantes que comparem o desempenho dessas crianças com as que apresentam alterações de linguagem, bem como a abrangência de mais faixas etárias.

\section{Referências bibliográficas}

1. Chomsky N. Novos horizontes no estudo da linguagem e da mente. São Paulo: Editora UNESP, 2005.

2. Demuth K, Machobane M, Moloi F. Rules and construction effects in learning the argument struture of verbs. J Child Lang. 2003; 30(4): 797-821.

3. Devescovi A, Caselli MC, Marchione D, Pasqualetti P, Reilly J, Bates E. A cross linguistic study of the relationship between grammar and lexical development. J Child Lang. 2005; 32(4): 759-86.

4. Befi-Lopes DM, Cáceres AM. Análise da diversidade de verbos enunciados na fala espontânea de pré-escolares brasileiros. Pró-Fono Rev. Atual. Cient. 2010; 22(1): 3-6

5. Araunachalam S, Waxman SR, Grammatical form and semantic context in verb learning. Lang Learn Dev. 2011; 7(1): $169-84$.

6. Gentner D. On relational meaning. The acquisition of verb meaning. Child development. 1978; 49: 988-98. 
7. Brancalioni AR, Zauza A, Karlisnki CD, Quitaiski LF, Thomaz, MFO. Desempenho do vocabulário expressivo de préescolares de 4 a 5 anos da rede pública e particular de ensino. Audiol Commun Res. 2018; 1-9.

8. Befi -Lopes DM, Cáceres AM, Araújo K. Aquisição de verbos em pré-escolares falantes do Português Brasileiro. Rev CEFAC. 2007; 9(4): 444-52.

9. Mobayyen F, Almeida RG. The influence of semantic and morphological complexity of verbs on sentence recall: implications for the nature of conceptual representation and category-specific deficits. Brain Cogn. 2005; 57(2): 168-71.

10. Gertner Y, Fisher C, Eisengart J. Learning words and rules: abstract knowledge of word order in early sentence comprehension. PsycholSci. 2006; 17(8): 684-91.

11. Felício CM, Ferreira CL. Protocol of orofacialmyofunctional evaluation with scores. Int J PediatrOtorhinolaryngol. 2008; 72(3): 367-75.

12. Hage SRV. Distúrbio específico do desenvolvimento da linguagem: subtipos e correlações neuroanatômicas. [Tese] Campinas (SP): Faculdade de Ciências Médicas - UNICAMP; 2000.

13. Zorzi JL, Hage SRV. PROC - Protocolo de observação comportamental: avaliação de linguagem e aspectos cognitivos infantis. São José dos Campos: Pulso Editorial; 2004.

14. Lidden G, Kankkonen A. Visual reinforcement audiometry. Acta Oto-Laryngologica. 1961; 67(2): 281-92.

15. Downs MP. Audição na infância. In: Northem JL, Downs MP. $5^{\text {a }}$ ed. Rio de Janeiro: Guanabara Koogan; 2005. p 129-167.
16. Shriberg LD, Kwiatkowski J, Hoffmann KA. A procedure for phonetic transcription by consensus. JSLHR. 1984; 27(3): 456-65.

17. McLeod S, Harrison LJ, McCormack J. The intelligibility in context scale: validity and reliability of a subjective rating measure. JSLHR. 2012; 55(2): 648-56.

18. Gleitman L, Gillette J. O papel da sintaxe na aprendizagem dos verbos. In: Fletcher P, Mcwhinney B, organizadores. Compêndio da linguagem da criança. Porto Alegre: Artes Médicas; 1997. p. 153-63.

19. Araujo K, Befi-Lopes DM. Extensão média do enunciado de crianças entre 2 e 4 anos de idade: diferenças no uso de palavras e morfemas. RevSocBrasFonoaudiol. 2004; 9(1): 156-63.

20. Sancassani M. Aquisição de verbos: uma questão de perspectiva sintática? ReVEL. 2012; 6(1): 27-61.

21. Behrens, H. How difficult are complex verbs? Evidence from German, Dutch, and English. Linguistics. 1998; 36(4): 679-713.

22. Kauschke C, HofmeisterC.Early lexical development in German: a study on vocabulary growth and vocabulary composition during the second and third year of life. J Child Lang. 2002; 29(4): 735-57.

23. Naigles LR, Hoff-Ginsberg E. Why are some verbs learned before other verbs? Effects of input frequency and structure on children's early verb use. J Child Lang. 1998; 25(1): 95-120.

24. Wiethan FM, Mota HB, Moraes AB. Correlações entre a produção de classes fonêmicas e classes gramaticais no Português Brasileiro. AudiolCommun Res. 2016; 21: e1669.

25. Andersen EML. Representações lexicais subjacentes: verbos e léxico inicial. Revel. 2008; 6(11): 1-31. 